\title{
Study on preprocessing in array detector based optical spatial filtering velocimetry
}

\author{
M. Schaeper, R. Kostbade, N. Damaschke \\ University of Rostock, Faculty of Computer Science and Electrical Engineering, \\ Institute of General Electrical Engineering, Optoelectronics and Photonic Systems \\ Albert-Einstein-Str. 2, 18059 Rostock, Germany \\ Martin.Schaeper@uni-rostock.de
}

\begin{abstract}
Spatial filtering velocimetry (SFV) is a technique for optical velocity measurements. In standard applications the image information of an observed scene is captured by a pixel-based array detector. The pixels' gray scale values are multiplied by a predefined grating function. By integrating over the weighted image information a signal point of the spatial filtering signal is generated for each frame. The spatial filtering signal is generated out of a sequence of images. The frequency of this alternating signal is proportional to the velocity of the observed scene.

The paper presents a Fourier-based description of spatial filtering velocimetry, which shows the relation to other displacement measurement techniques like cross correlation or auto correlation methods used in particle image velocimetry (PIV). The spatial filtering signal can be interpreted as a single temporal changing Fourier coefficient. The spatial filtering signal is disturbed by phase jumps. They occur, when the statistical spectral content of the observed scene is temporal changing. The fluctuations of the amplitude and the signal's phase have a negative impact on the frequency estimation. Our approach is a specific preprocessing of the intensity distribution of the array detector to optimize the spatial filtering signal. Here the incoming and outgoing patterns of the image information are suppressed. So the spectral content is sectional stabilized and the timestamps of spectral changes are known.
\end{abstract}

Key words: spatial filtering, cross correlation, Fourier coefficient, phase jumps, stabilization

\section{Introduction}

In many processes the measurement of the velocity has an important role. Length and acceleration can be derived from the measured velocity as additional process factors.

Besides laser based imaging techniques and laser Doppler velocimetry, spatial filtering velocimetry (SFV) is a measurement technique for velocity measurements on solid surfaces and in fluid flows [1] with a number of advantages. The main advantages are: the use of incoherent light sources and the reduced complexity of the signal processing. For the last decades SFV was implemented and applied to a variety of problems. The application of SFV to particle sizing using a fibre-optical probe is described by Petrak et al [2]. Hosokawa et al described the use of SFV applied on image series for tomographic fluid flow measurements [3]. A recent development is the application of SFV for the detection of rotational movement of rotating biological cells [4].
In this study we focus on the reduction of the signal data to perform real time applications and on a description for determine the validity of the measurement results. Both approaches are preprocessing steps. A mathematical description is given first to illustrate how the spatial filtering signal is generated.

\section{Theory of spatial filtering velocimetry}

When using array detectors the signal generation can be described as follows. The observed moving structure is imaged by an object lens onto an array detector (see Fig. 1). The moving image information $i(x-v t)$ is given as pixel gray values and is weighted by a grating function $g(x)$ as:

$$
s(t)=\int_{-\infty}^{+\infty} i(x-v t) g(x) \mathrm{d} x
$$

The grating function can be arbitrary. Ideal grating functions contain only one frequency. 
We recommend sinusoidal shaped grating functions as:

$$
\underline{g}(x)=\cos (2 \pi \mu x)-\mathrm{j} \sin (2 \pi \mu x)=e^{-\mathrm{j}(2 \pi \mu x)}
$$

The grating function with spatial frequency $\mu$ (compare to Aizu and Asakura [5]) is complex to get the directional information of the structure's movement. The angular spatial frequency $k$ can be found as:

$$
k=2 \pi \mu \text {. }
$$

A cosine shaped grating function is given in Fig. 1 as an example. Please note that discrete pixel values $n_{P}$ are used for the grating function $g_{n_{p}}$.

By using a complex grating function the calculation of the signal of eq. (1) can be written as:

$$
\underline{s}(t)=\int_{-\infty}^{+\infty} i(x-v t) e^{-j(2 \pi \mu x)} \mathrm{d} x=F T\{i(x-v t)\}_{\mu}(4)
$$

where we can find, that the spatial filtering signal corresponds to a time dependent Fourier (FT-Fourier Transform) coefficient.

In comparison to other imaging techniques (e.g. particle image velocimetry-PIV) often the crosscorrelation or auto-correlation are used. For a reduced amount of calculation it is common to use the Fourier transformation of the captured images $I(k)$. Then the spatial spectra of consecutive images are multiplied (conjugatecomplex). The result of the cross-correlation function (CCF) can be obtained by back transformation as:

$$
C C F=F T^{-1}\left\{I_{n-1}^{*} I_{n}\right\} .
$$

Here we can see that the cross-correlation uses all coefficients of the Fourier transformation for estimation of the displacement.

\section{Data generation}

The experimental data were taken using a digital signal processor (DSP) based measurement system. The sensor is an optical position sensor [6], which sums up pixel columns and pixel rows inside of the chip. Therefore much higher frame rates can be reached (see preprocessing by summation of pixel columns) $A$ description of the whole measurement system is given in [7]. The raw data, shown here, were collected at a frame of $3200 \mathrm{fps}$ and 8 bit resolution.

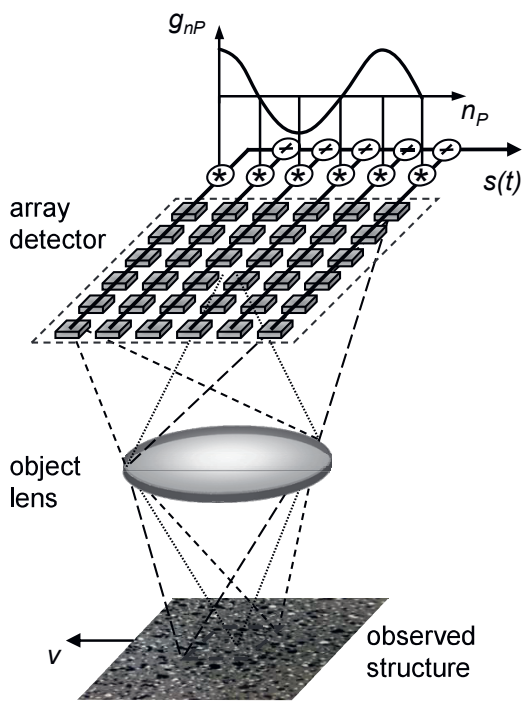

Fig. 1. Principle of spatial filtering velocimetry using array detectors

The observed moving process was a surface of a conveyor-belt with a stochastic structure (as shown in Fig. 2 in the upper right corner). A rotational encoder was installed at the second pulley for referencing the movement. By using this experimental set-up the displacement can be referenced to an accuracy of $0.06 \%$ of the local displacement of the observed structure.

The experimental raw data were stored and analyzed offline. The following analysis compares the results of the classical spatial filtering with the result using preprocessing for stabilization of Fourier coefficients (see section Preprocessing for detection of phase jumps).

\section{Preprocessing by summation of pixel columns}

The first preprocessing step is the summation of pixel columns, as shown in Fig. 1. As described in [7] the summation significantly reduces the amount of data from $n \times n$ down to $n$ values. For example the pixels' gray values of an area of $256 \times 256$ pixels is reduced to 256 sum values. This data reduction is already implemented on the used smart pixel sensor. Therefore, frame rates in the region of $\mathrm{kHz}$ are possible [6].

Beside the scope of this article a further option for preprocessing in SFV is known. It is possible to use a micro mirror array in the optical path. With the tilt of the micro mirrors $(+1$ and -1$)$ the image information of the observed structure can be directly optically weighted by the tilt of the micro mirrors. Afterwards the weighted image information can be integrated optically by a lens onto a single photo detector as described in [8]. The measurement rate is therefore limited by the photo diode, typically in the $\mathrm{MHz}$ region. 
Preprocessing for detection of phase jumps

A measurement system sees a limited spatial section of the whole observed structure only. During the measurement a number of information moves into the observation area and other information moves out of this area. This results in phase jumps in the spatial filtering signal.

Up to now, phase jumps where repressed by using a plausibility check. For this, the current measurement value was compared with prevenient measurement results. Another approach is the use of the spatial filtering signal's magnitude. High amplitudes are indicating high signal to noise ratios. The disadvantage is that a high number of measurements are discarded, although they could be useful.

Our approach detects dominant structures entering and leaving the observed region. An illustration of this preprocessing is given in Fig. 2. The gray line (Fig. 2) is indicating the original sum signal of the sensor in $x$-direction. The values are normalized by 255 ( 8 bit resolution). An example picture of the observed surface is shown in the upper right corner of Fig. 2. With the help of a threshold, the dominant structure can be extracted (black dashed line). Every structure crossing the threshold at the border of the image is taken into account for further signal processing. The other structures at the border are set to the value of the threshold.

The level of the threshold depends on the observed process. For structured surfaces, as shown in the upper right corner of Fig. 2, the mean value of the original signal is an acceptable value for a threshold. In the case of particle image series, i.e. observed scenes with low density of particles, the mean value would be very low, probably in the noise level of the image. Here a threshold between maximum and minimum is advisable. To cover both cases we suggest the mean of both values (mean signal value and mean of maximum and minimum) Additionally the use of a threshold band is recommended for suppressing noise.

With our approach the phase jumps are located at known time stamps. Therefore the signal values at this timestamps can be rejected from the statistics, and the other signal values are not disturbed by phase jumps and are more reliable.

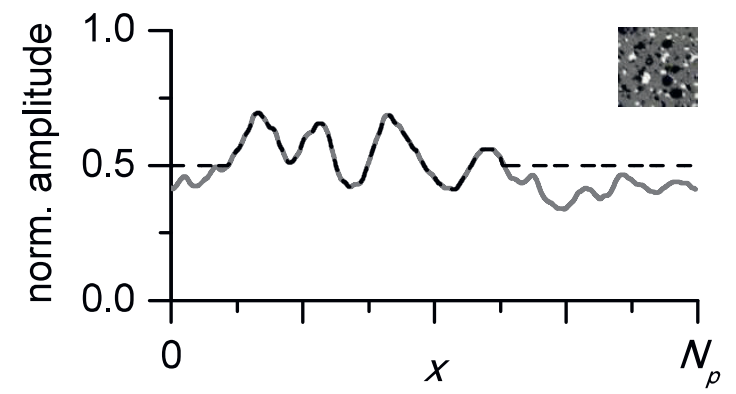

Fig. 2. Example for preprocessing using a threshold to avoid stochastic phase jumps. gray line: original sum values; dashed black line: preprocessed data by applying a threshold.

\section{Results and discussion}

In Fig. 3 the effect of the described preprocessing is demonstrated as comparison between an original signal section and the preprocessed section. The signals are normalized to 255 (8 bit resolution). The original spatial filtering signal (Fig. 3a), real part, gray solid line) has a non-continuous alternating characteristic. The magnitude of the complex signal (dotted line) is not stable and changes temporally. The phase of the original signal (Fig. 3c, gray solid line) diverges locally from the expected trend. Especially at $0.15 \mathrm{~s}$ the phase's gradient is showing an incorrect movement of the observed scene in the opposite direction. In contrast to that the preprocessed signal of the same section is shown in Fig. 3b (real part, black dashed line). The detected and therewith induced phase jumps are indicated by light gray vertical lines. Between the detected phase jumps the signal shows a stable linear phase slope because the spectral content of the observed scene is constant. Especially the magnitude of the complex signal (dotted line) is now constant in sections between two detected phase jumps. This leads to ideal signals between the induced phase jumps. The phase of the signal from the preprocessing is additionally shown in Fig. 3c (black dashed line) for comparison. In contrast to the original signal the phase shows the expected trend between the phase jumps.

Result of the presented approach is a reduction of the measurement uncertainty to a quarter of the original signal analysis. 


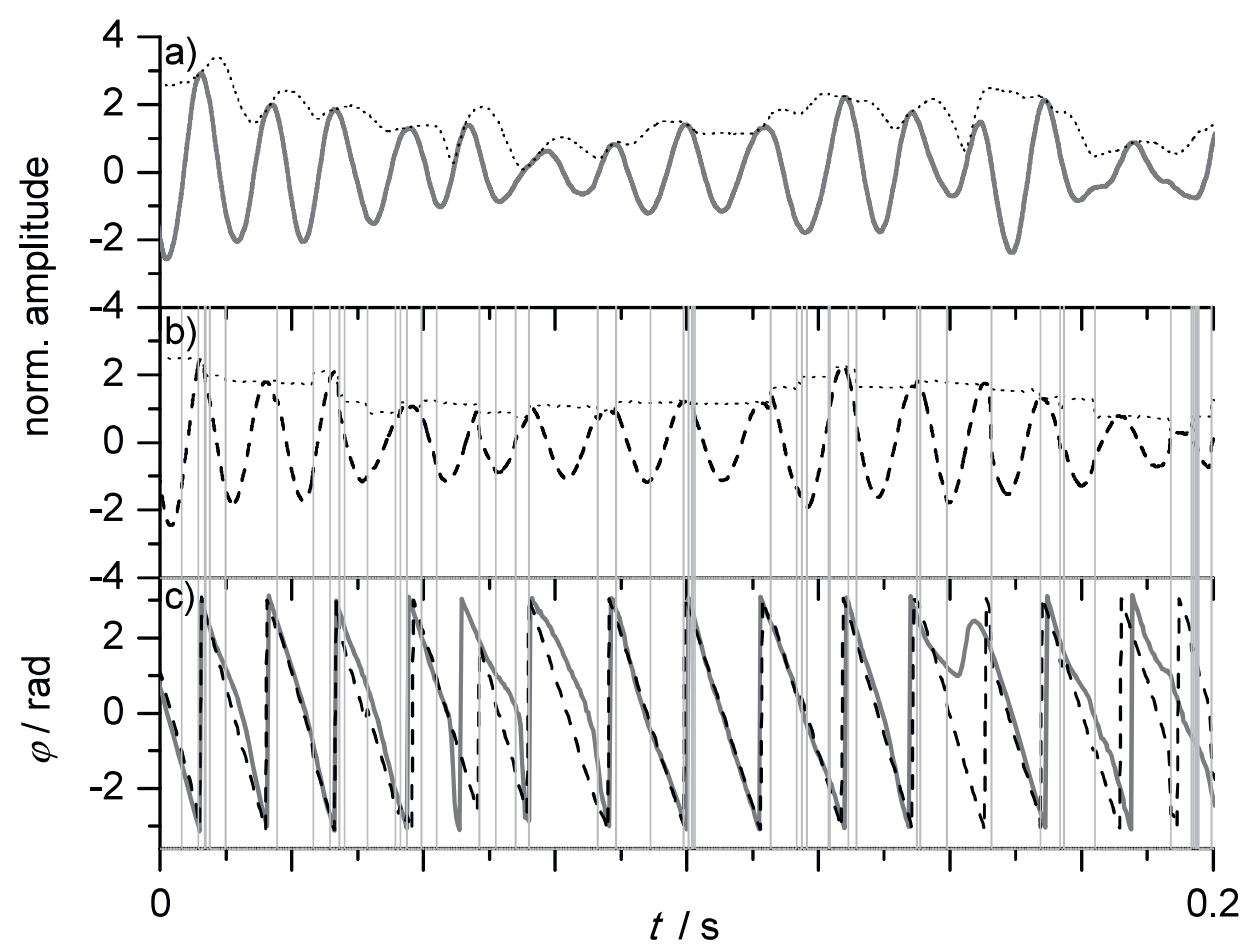

Fig.3. Comparison of original SFV signal (in a) gray solid line) and the additional use of a data preprocessing to avoid stochastic phase jumps (in b) black dashed line). The magnitudes of the complex signals are indicated as black doted lines (a) and b)). The comparison of the signals' phases is shown in c). Detected phase jumps are indicated as gray vertical lines (b) and c)).

\section{Conclusion}

In this paper two possibilities of preprocessing using array detectors are presented. The first is a pure summation of the pixels' columns to reduce the amount of data and to increase the frame rate of the measurement system. A higher frame rate denotes a higher number of measurement results which leads to an improved accuracy.

In optical velocity measurements the sensor has a finite view on an observed scene. Significant structures are moving in and out of the field of view. In SFV this results in phase jumps, which cannot be detected in the spatial filtering signal. The signal's phase diverges in the temporal vicinity of the phase jumps from the expected linear trend. A low pass filtering at the image border does not solve this problem. Another possibility is a plausibility check of the current measurement result. The disadvantage is that also reliable measurement values would be discarded. For this reason the raw signals, i.e. the sum values of the sensor, have to be observed. Our approach is to use a threshold based detection of incoming and leaving structures in the sensor's raw data. The phase jumps are detected and the time stamp of the phase jumps are saved. Every time, when a phase jump occurs, the actual measurement result has to be rejected. This localization of phase jumps to discrete and known time stamps leads to a higher number of useful measurement results and increases the accuracy.

A fact that has to be mentioned is that the movement of the observed scene has to be in the direction of the spatial filtering structure. The presented preprocessing observes the images border only. If the spatial filter is not aligned to the velocity direction, structures moving transverse to the spatial filter would produce additional phase jumps. This would not be detectable by the presented preprocessing. The solution is to align the spatial filter to the moving direction. When using array detectors the direction of the spatial filtering structure, i.e. the direction of the grating function, can be adapted by software.

Both the preprocessing by summation of pixel columns and the preprocessing for detection of stochastic phase jumps help to improve accuracy of the velocity measurement. 


\section{References}

[1] Y. Aizu, T. Asakura, Spatial Filtering Velocimetry Fundamentals and Applications, Optical Sciences, Springer Science \& Business, Berlin Heidelberg (2006); doi: 10.1007/3-540-28215-7

[2] D. Petrak, S. Dietrich, G. Eckardt, M. Köhler, Inline particle sizing for real-time process control by fibre-optical spatial filtering technique (SFT), Advanced Powder Technology 22(2), p. 203-208 (2011); doi: 10.1016/j.apt.2010.11.002

[3] S. Hosokawa, T. Matsumoto, A. Tomiyama, Tomographic Spatial Filter Velocimetry for ThreeDimensional Measurement of Fluid Velocity, 16th Int. Symp. on Applications of Laser Techniques to Fluid Mechanics Lisbon, Portugal (2012)

[4] M. Schaeper, R. Schmidt, R Kostbade, N. Damaschke, J. Gimsa, Optical high-resolution analysis of rotational movement: testing circular spatial filter velocimetry (CSFV) with rotating biological cells, Journal of Physics D: Applied Physics 49(26) 265402 (2016); doi: $10.1088 / 0022-3727 / 49 / 26 / 265402$
[5] Y. Aizu, T. Asakura, Principles and Development of Spatial Filtering Velocimetry, Applied Physics B: Lasers and Optics 43(4) 209-224 (1987); doi: 10.1007/BF00692491

[6] Hamamatsu, Profile Sensor S9132, High-Speed frame rate Sensor capable of acquiring twodimensional projection data, Hamamatsu Photonics K.K. Solid State Division (2004)

[7] M. Schaeper, N. Damaschke, Velocity Measurement for Moving Surfaces by Using Spatial Filtering Technique Based on Array Detectors, Autonomous and Intelligent Systems, Editors: M. Kamel, F. Karray, W. Gueaieb, A. Khamis, Springer Berlin / Heidelberg: Burnaby, BC, Canada, 303-310 (2011) doi: 10.1007/978-3642-21538-4_30

[8] M. Degner, H. Krüger and H. Ewald. Micromirror array based optical spatial filter technique for fast and flexible velocimetry. Instrumentation and Measurement Technology Conference (I2MTC) Proceedings, IEEE International (2014) 